\title{
Exploring Visible Spectrum: A Study of Light Fidelity System and Its Applications
}

\author{
Deepti Rai ${ }^{1}$, Monika Shah ${ }^{2}$ \\ ${ }^{1}$ Proffessor Electronics \& Comm. Engg. Dept., Alpine Institute of Technology, Ujjain (M.P) \\ ${ }^{2}$ PG Student, Department of Electronics and Communication Engineering, Alpine Institute of Technology, Ujjain (M.P)
}

\begin{abstract}
This Document gives an introductory analysis of how Li Fi technology can provide a wide range of spectrum to the user with very low interference. It also gives the details of the technology and its advantages over the contemporary Wi-Fi technology.Morever since we are using the spectrum at a rapid rate this paper also shows the potential viability of light fidelity system as a major promising source of technology for tomorrow.
\end{abstract}

Keywords: VLC, LED bulb, fast flickering lights , LI-Fi router.

\section{Introduction}

$\mathrm{Li}-\mathrm{Fi}$ is the term some have used to label the fast and cheap wireless-communication system, which is the optical version of Wi-Fi. The term was first used in this context by Harald Haas in his TED Global talk on Visible Light Communication. The technology was demonstrated at the 2012 Consumer Electronics Show in Las Vegas using a pair of Casio smartphones to exchange data using light of varying intensity given off from their screens, detectable at a distance of up to 10 metres. Also known as Visible Light Communication (VLC), it offers a real alternative to radio based communications. The spectrum is free, plentiful, and the cost of implementation is actually less than equivalent radio technology. This technology also saves a lot of energy.

\section{Experimental Proof}

Harald Haas demonstrated his invention using an ordinary table lamp that successfully transmitted data at speeds exceeding $10 \mathrm{Mbps}$ using light waves from LED light bulbs to a computer located below the lamp. To prove that the light bulb was the source of the data stream, he periodically blocked the beam of light, causing the connection to drop. Here's how Haas describes the technology: "replace these inefficient incandescent light bulbs, florescent lights, with this new technology of LED, LED light bulbs. An LED is a semiconductor. It's an electronic device. And it has a very nice acute property. Its intensity can be modulated at very high speeds, and it can be switched off at very high speeds. And this is a fundamental basic property that we explored with our technology. . . . We transmit with our technology, not only a single data stream, we transmit thousands of data streams in parallel, at even higher speeds. And the technology we have developed - it's called SIM OFDM. And it's spacial modulation - these are the only technical terms, I'm not going into details - but this is how we enabled that light source to transmit data." The big vision: One day we might be able to use light to access the Internet in addition to - or even instead of - radio waves. Microchips in street lights and car headlights and lights in our home would give us wireless data transmission right along with illumination ${ }^{[1]}$.

\section{How does it Work}

- LEDs are used as the source of light. For processing the data LED holds a micro-chip

- Basic Principle behind the working of this technology is "if the LED is on, you transmit a digital 1, if it's off you transmit a 0". This is how the data is transmitted through this technique. It looks like a fast flickering of light.

- It is possible to encode data in the light by varying the rate at which the LEDs flicker on and off to give different strings of $1 \mathrm{~s}$ and $0 \mathrm{~s}$. The LED intensity is modulated so rapidly that human eyes cannot notice.

- Parallel data is sent though the LEDs where each LED transmits a single data stream.

\section{Why LEDs?}

To turn a light into a Li-Fi router involves modulating its output, to carry a message, and linking it with a network cable to a modem that is connected to a telephone or cable-broadband service, just like a Wi-Fi router. Incandescent light bulbs and fluorescent tubes are not really suitable for modulation, but they are yesterday's lighting technology. Tomorrow's is the light-emitting diode. LEDs are rapidly replacing bulbs and tubes because they are more efficient. And because they are semiconductor devices, tinkering with their electronics to produce the flickering signals required for data transmission is pretty straightforward $^{[2]}$.

\section{A. Following is a comparison between the LI-Fi and the} conventional Wi-Fi technologies:

Table I: A Comparison between the two technologies

\begin{tabular}{|c|c|c|}
\hline Technology: & Li Fi & Wi Fi \\
Transmission medium: & Visible Light & Electromagnetic waves \\
Bandwidth & Typically in GHz & Typically in MHz \\
Line of sight & Yes & No \\
\hline
\end{tabular}




\section{International Journal of Science and Research (IJSR) \\ ISSN (Online): 2319-7064}

Index Copernicus Value (2016): 79.57 | Impact Factor (2015): 6.391

\section{B. Applications}

- Medical Field: Operating rooms do not allow Wi-Fi over radiation concerns, and there is also that whole lack of dedicated spectrum. While $\mathrm{Wi}-\mathrm{Fi}$ is in place in many hospitals, interference from cell phones and computers can block signals from monitoring equipment. Li-Fi solves both problems: lights are not only allowed in operating rooms, but tend to be the most glaring (pun intended) fixtures in the room.

- Traffic Control and Security: Sensors implanted in your front and rear bumpers could receive data transmitted from the rear lights of that car that just veered into your lane while you were texting. Both drivers are warned (or maybe the car takes over) and the accident is averted. This can also work with traffic lights, possibly sending your car info about road conditions, warning you about that guy you can't see speeding toward the intersection, or instantly transmitting his plate number to the cops when he does.

- Offices and Institutes: A very high speed connection (say $100 \mathrm{Mbps}$ for everyone) of Internet can be provided to the employees in an office or to the students in an institute

- Apart from these applications Li-Fi can also be used for communication in underwater operations, in power plant and other industries, in smart classes, in aircraft for data transmission.

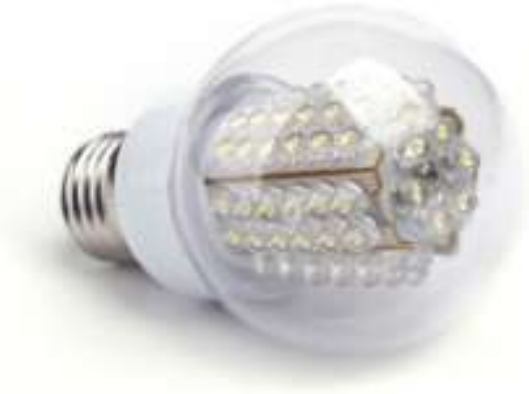

Figure 1: An LED bulb that work as a Li-Fi adaptor

\section{Speed and mode of operation}

Li-Fi, as it has been dubbed ${ }^{[3]}$, has already achieved blisteringly high speeds in the lab. Researchers at the Heinrich Hertz Institute in Berlin, Germany, have reached data rates of over 500 megabytes per second using a standard white-light LED. Haas has set up a spin-off firm to sell a consumer VLC transmitter that is due for launch next year. It is capable of transmitting data at $100 \mathrm{MB} / \mathrm{s}$ - faster than most UK broadband connections. In October 2011 a number of companies and industry groups formed the Li-Fi Consortium $^{[4]}$, to promote high-speed optical wireless systems and to overcome the limited amount of radio-based wireless spectrum available by exploiting a completely different part of the electromagnetic spectrum. The consortium believes it is possible to achieve more than $10 \mathrm{Gbps}$, theoretically allowing a high-definition film to be downloaded in 30 seconds.

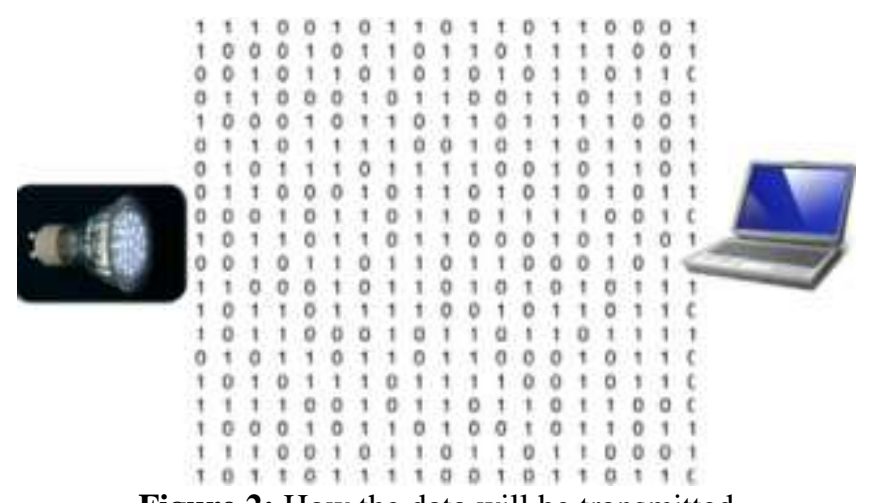

Figure 2: How the data will be transmitted

Once established, VLC could solve some major communication problems. In 2009, the US Federal Communications Commission warned of a looming spectrum crisis: because our mobile devices are so data-hungry we will soon run out of radio-frequency bandwidth. Li-Fi could free up bandwidth, especially as much of the infrastructure is already in place ${ }^{[5]}$. More sophisticated techniques could dramatically increase VLC data rates. Teams at the University of Oxford and the University of Edinburgh are focusing on parallel data transmission using arrays of LEDs, where each LED transmits a different data stream. Other groups are using mixtures of red, green and blue LEDs to alter the light's frequency, with each frequency encoding a different data channel ${ }^{[6]}$.

Because it uses light rather than radio-frequency signals, VLC could be used safely in aircraft, integrated into medical devices and hospitals where Wi-Fi is banned, or even underwater, where Wi-Fi doesn't work at all.

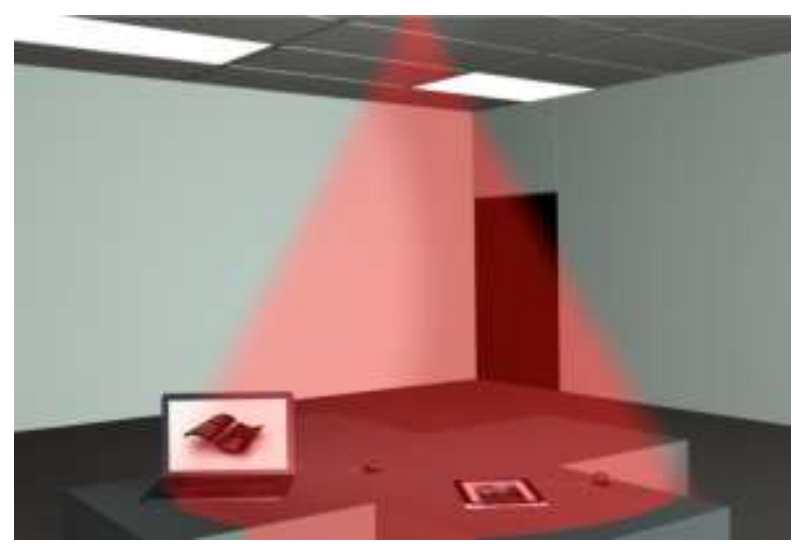

Figure 3: The GigaShower

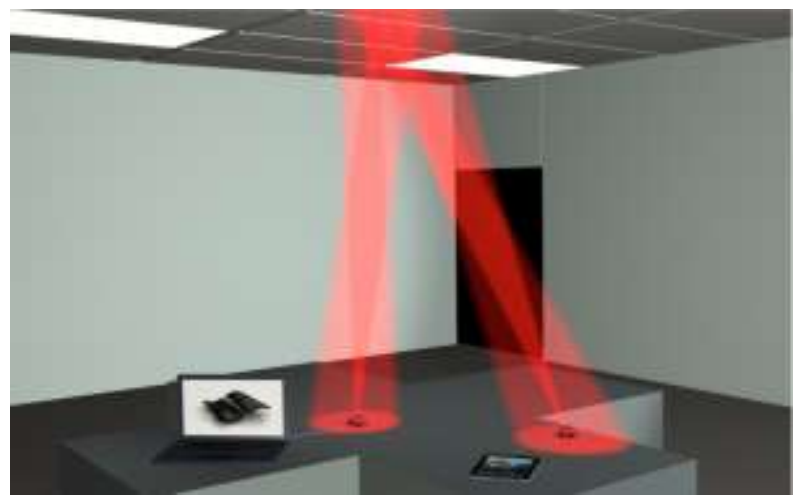

Figure 4: The GigaMIMO

Volume 6 Issue 12, December 2017

www.ijsr.net

Licensed Under Creative Commons Attribution CC BY 


\section{Conclusions}

Though there are some limitations too. Unlike radio, light waves will not penetrate walls. Yet for secure applications that could be a bonus. And light bulbs - some 14 billion of them around the world - are almost everywhere and often on. As they are gradually replaced by LEDs ${ }^{[7]}$, every home, office, public building and even streetlight could become a $\mathrm{Li}-\mathrm{Fi}$ hotspot. Having a line-of-sight connection with the LED in question would undoubtedly improve the signal, but light reflected from walls or ceilings might often be enough. In any case, having a good line of sight helps Wi-Fi as well. And spotting a nearby light in order to sit next to it is certainly easier than finding the location of a Wi-Fi router.

\section{References}

[1] http://www.lificonsortium.org..

[2] http://www.newscientist.com/article/mg21128225.400-w ill-lifi-be-the-new-wifi.html.

[3] http://marginalrevolution.com/marginalrevolution/2011/ 08/new-wireless-technologies-lifi-and-dido.html.

[4] http://www.luxim.com/.

[5] Haas, Harald (July 2011). "Wireless data from every light bulb". TED Global. Edinburgh, Scotland.

[6] Tony Smith (24 May 2012). "WTF is... Li-Fi? Optical data transfer's new leading light?". The Register. Retrieved 22 October 2013.

[7] An IEEE Standard for Visible Light Communicationsvisiblelightcomm.com, dated April 2011.Tsonev, D.; Sinanovic, S.; Haas, Harald (15 September 2013). "Complete Modeling of Nonlinear Distortion in OFDM-Based

[8] Optical Wireless Communication". IEEE Journal of Lightwave $\quad$ Technology 31 (18): $3064-$ 3076.doi:10.1109/JLT.2013.2278675 